\title{
Impact of COVID-19 in gynaecological oncology care: a systematic rapid review
}

\author{
Manolis Nikolopoulos ${ }^{1}$ - Manish K. Maheshwari ${ }^{1} \cdot$ Stergios K. Doumouchtsis . $^{1,2,3,4,5}$. CHORUS: An International \\ Collaboration for Harmonising Outcomes, Research and Standards in Urogynaecology and Women's Health \\ (https://i-chorus.org/)
}

Received: 14 May 2021 / Accepted: 13 October 2021 / Published online: 3 November 2021

(c) The Author(s), under exclusive licence to Springer-Verlag GmbH Germany, part of Springer Nature 2021

\begin{abstract}
Purpose Synthesis of available evidence on clinical practice in gynaecological oncology during the COVID-19 pandemic is highly warranted, as women with cancer are at increased risk due to their systemic immunosuppressed state and changes in their care are inevitable. Rapid review of available data is a quick way of providing useful information and insight into the way medical practice has been affected by the COVID pandemic.

Methods We conducted a systematic rapid review, based on a literature search of MEDLINE/PubMed, Embase, and Cochrane CENTRAL. We considered all studies on gynaecological oncology care during the COVID-19 pandemic using relevant keywords and MeSH terms. Selection criteria were English language, studies with more than five cases and publication in peer-review journal.

Results Nine retrospective studies, one systematic review and five questionnaire surveys were included. Quality of the studies has been assessed. Development of higher quality evidence is warranted. Mortality of COVID-19 infection is higher in patients with gynaecological cancer than in non-cancer patients. Reported delays in diagnosis and management of cancer and changes in treatments, may affect the natural history of cancer and increase patients' anxiety and fear of disease progression while causing concerns to healthcare professionals affecting their clinical practice. The number of new diagnoses has declined. Prioritization is important, face-to-face interactions should be limited, and appropriate protective measures are essential. Cancer surgery, chemotherapy, and radiotherapy should continue as high priority practices.

Conclusion The COVID-19 pandemic has affected clinical practice significantly. Adaptations in clinical practice may improve mortality and complication rates.
\end{abstract}

Keywords Gynecology · COVID-19 · Surgical oncology · Surgery $\cdot$ Uterine cancer

Manolis Nikolopoulos

manolisnikolopoulos@gmail.com;

manolis.nikolopoulos@nhs.net

1 Department of Obstetrics and Gynecology, Epsom and St Helier University Hospitals NHS Trust, Epsom, UK

2 St George's University of London, London, UK

3 Laboratory of Experimental Surgery and Surgical Research N S Christeas, University of Athens, Medical school, Athens, Greece

4 School of Medicine, American University of the Caribbean, Pembroke Pines, FL, USA

5 School of Medicine, Ross University, Miramar, FL, USA

\section{Introduction}

Almost 100 million cases of coronavirus have been detected worldwide with 2.363 .844 deaths in 220 countries until February 2021 [1]. Many scientific societies have issued recommendations for the treatment of specific pathologies, including societies related to gynaecological oncology and gynaecological surgery. These recommendations have been made mainly on learned assumptions or expert opinions [2].

Many medical centers have not been able to offer gynaecological cancer surgery with optimal guarantees of health and safety for patients and staff. This has resulted in recommendations of non-surgical alternatives to standard treatment during the early stages of the pandemic [3]. Early reports suggest that people with cancer may experience 
worse outcomes from COVID-19, including higher risk of admission to intensive care units, requirement for invasive ventilation and death [4].

Another significant issue is the delay in diagnosis and treatment, which are advocated for controlling the progression of cancer. The stage of cancer is the most crucial factor in prognosis, survival, recurrence rate, and in the choice of treatment. However, no systematic review has reached a conclusive agreement regarding the effect of treatment delay in different types of cancer [5].

In some circumstances, there is a need to accelerate the review process and especially during the COVID-19 pandemic, it is essential to provide quick evidence to allow changes and recommendations to develop [6]. Rapid reviews are, therefore, highly warranted, even if certain time consuming steps, which are usually part of a systematic review process, are omitted [7]. Although literature is full of expert opinions addressing the potential impact of COVID-19 on oncologic practice [1], only few data on the impact of COVID-19 on patients affected by cancer are still available [8].

The aim of this systematic rapid review was to evaluate the impact of the COVID-19 pandemic on patients with gynaecological cancer and the impact of cancer on the clinical presentation of COVID-19 infection. We also aimed to summarize clinical practice recommendations to facilitate harmonization of practices and optimization of outcomes across different clinical settings.

\section{Material and methods}

A systematic rapid review was conducted in accordance with the Preferred Reporting Items for Systematic Reviews and Meta-Analyses (PRISMA) guidelines and the guidance for rapid reviews published by Cochrane [7]. The rapid review's research questions and methods, including search strategy and inclusion criteria, were pre-defined in a research protocol document. This study was undertaken by a working group led by CHORUS: An International Collaboration for Harmonising Outcomes, Research, and Standards in Urogynaecology and Women's Health (https://i-chorus.org/), applying similar methodology standards to a recently published rapid review in urogynaecological practice [9]. Our methodology has been adapted from recent methodological guidance published by Cochrane [7].

Systematic searches of biomedical databases were conducted (MEDLINE via PubMed, Embase, and Cochrane CENTRAL) on 28th of September 2020 and updated on 10th of February 2021. One reviewer used a combination of terms relating to COVID-19 epidemic or pandemic (COVID-19, SARS-COV-2, Coronavirus) and gynaecological cancer, including terms: endometrial, cervical, ovarian, and vulval cancer with variant spelling. Articles were extracted and a second reviewer verified the results. PICO elements were used. Inclusion criteria were: English language, studies with more than five reported cases and publication in a peerreview journal.

Prospective and retrospective studies were included reporting:

- The impact of COVID-19 infection in patients with existing gynaecological cancer and immunosuppression,

- The effect of the coronavirus pandemic in the practice of gynaecological oncology and in gynaecological oncologic centers, with special reference to the impact on the cancer diagnoses and management plans,

- The physical and psychological effect of the pandemic on patients with gynaecological cancer and healthcare personnel.

No date limits or methodological filters were applied to the searches.

Results are presented in narrative summary using supplementary flowchart and tables.

The quality of the retrospective studies was evaluated using the Newcastle-Ottawa Scale [10]. The quality of the online studies was not assessed.

\section{Results}

\section{Summary of evidence}

A total of 335 citations were identified in the literature search. Following screening of titles and abstracts, 29 potentially relevant reports were retrieved for full-text review. Of these articles, three articles were excluded for being case reports and 15 publications met the inclusion criteria and were included in this study (Fig. 1).

\section{Study design and data collection}

One systematic review was identified. Nine studies were retrospective reviews of cases and five studies were questionnaire surveys; three of them addressed to doctors and two to patients. Overall quality assessment scores can be seen in Table 1 with " 1 " being the lowest score and "9" the highest. It is apparent that studies of higher quality are needed.

\section{Country of origin}

Of the 14 studies, three were conducted in the USA, two in China and one in each of the following countries: Turkey, Italy, Spain, India, and Austria. The online studies were conducted in USA (two), India, and two of them globally. 


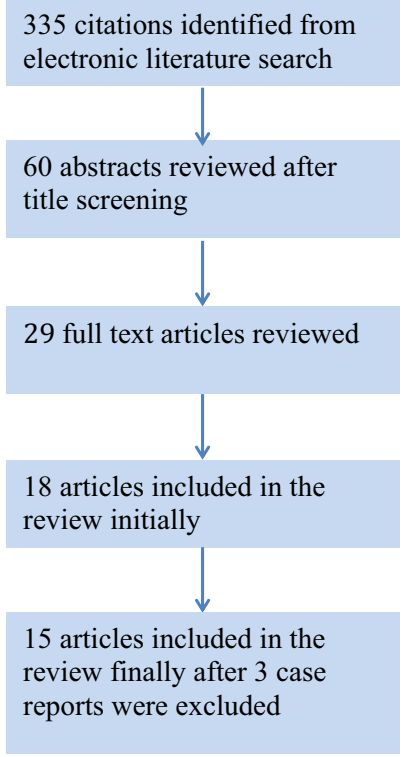

Fig.1 Selection process flowchart

\section{Summary of findings}

\section{Severity of COVID-19 infection in patients with gynaecological cancer}

Patients with cancer are considered to be at increased risk of COVID infection due to their systemic immunosuppressed state [4].

Five studies were identified that mentioned outcomes of COVID-19 infection in patients with gynaecological cancer [8, 11-14].
In a review of patients with gynaecological cancer that were infected with COVID-19 in New York hospitals, the mortality rate was $14 \%, 54.5 \%$ of patients required hospitalization and sadly none of the patients who were intubated recovered. No association was found between the timing of other forms of treatment and specifically lymphopenia was proved not to be associated with disease severity. Continuation of cancer treatment was considered safe and was strongly recommended [12] (Table 2).

Mortality rate was similar (13.5\%) in the study by Bogani et al. which included 355 patients admitted to the hospital for surgery or chemotherapy as treatment of gynaecological cancers [8].

Mehta et al. identified 218 cancer patients admitted with active COVID-19 infection with mortality rate of $25 \%$ and highest rate of mortality in patients with lung cancer of $55 \%$. Mortality among patients with gynaecological cancer was $38 \%$ [13].

Finally, six patients were identified with cervical cancer and active COVID-19 infection in a multicenter study in China with no deaths reported [11]. A study from the same region identified nine patients with cervical cancer and active infection with no deaths reported [14]. It is possible that these two studies have included duplicate data.

\section{Impact of COVID-19 pandemic on diagnosis and management of patients with gynaecological cancer}

Two studies reviewed the diagnostic process of gynaecological cancer during the pandemic and two further evaluated the impact of the pandemic on cancer treatment (Table 3).

Table 1 Detailed Newcastle-Ottawa Scale of each included cohort study

\begin{tabular}{|c|c|c|c|c|c|c|c|c|c|}
\hline Study & $\begin{array}{l}\text { Representa- } \\
\text { tiveness of } \\
\text { exposed } \\
\text { cohort }\end{array}$ & $\begin{array}{l}\text { Selection of } \\
\text { non-exposed } \\
\text { cohort }\end{array}$ & $\begin{array}{l}\text { Ascertain- } \\
\text { ment of } \\
\text { exposure }\end{array}$ & $\begin{array}{l}\text { Demonstra- } \\
\text { tion that } \\
\text { outcome of } \\
\text { interest was } \\
\text { not present at } \\
\text { start of study }\end{array}$ & $\begin{array}{l}\text { Comparabil- } \\
\text { ity of cohorts } \\
\text { on the basis } \\
\text { of the design } \\
\text { or analysis }\end{array}$ & $\begin{array}{l}\text { Assessment } \\
\text { of outcome }\end{array}$ & $\begin{array}{l}\text { Was follow- } \\
\text { up long } \\
\text { enough for } \\
\text { outcomes to } \\
\text { occur }\end{array}$ & $\begin{array}{l}\text { Adequacy of } \\
\text { follow-up of } \\
\text { cohorts }\end{array}$ & $\begin{array}{l}\text { Total } \\
\text { quality } \\
\text { score }\end{array}$ \\
\hline Lara et al. & 1 & 0 & 1 & 1 & 1 & 1 & 0 & 1 & 6 \\
\hline Bogani et al. & 0 & 0 & 1 & 1 & 0 & 1 & 0 & 1 & 4 \\
\hline Mehta et al. & 1 & 0 & 1 & 1 & 1 & 1 & 1 & 1 & 7 \\
\hline Dai et al. & 1 & 1 & 0 & 1 & 2 & 1 & 0 & 1 & 7 \\
\hline Yang et al. & 1 & 0 & 1 & 1 & 2 & 1 & 0 & 1 & 7 \\
\hline $\begin{array}{l}\text { Suh-Burg- } \\
\text { mann et al. }\end{array}$ & 1 & 0 & 1 & 1 & 0 & 1 & 0 & 0 & 3 \\
\hline $\begin{array}{l}\text { Tsibulak } \\
\text { et al. }\end{array}$ & 1 & 1 & 0 & 1 & 1 & 1 & 0 & 1 & 6 \\
\hline Dursun et al. & 1 & 0 & 0 & 1 & 0 & 1 & 1 & 1 & 5 \\
\hline $\begin{array}{l}\text { De Santiago } \\
\text { et al. }\end{array}$ & 1 & 0 & 0 & 1 & 0 & 1 & 1 & 0 & 4 \\
\hline
\end{tabular}

Score $0-1$ for selection and outcome categories. Score 0-2 for comparability. Maximum score: 9 


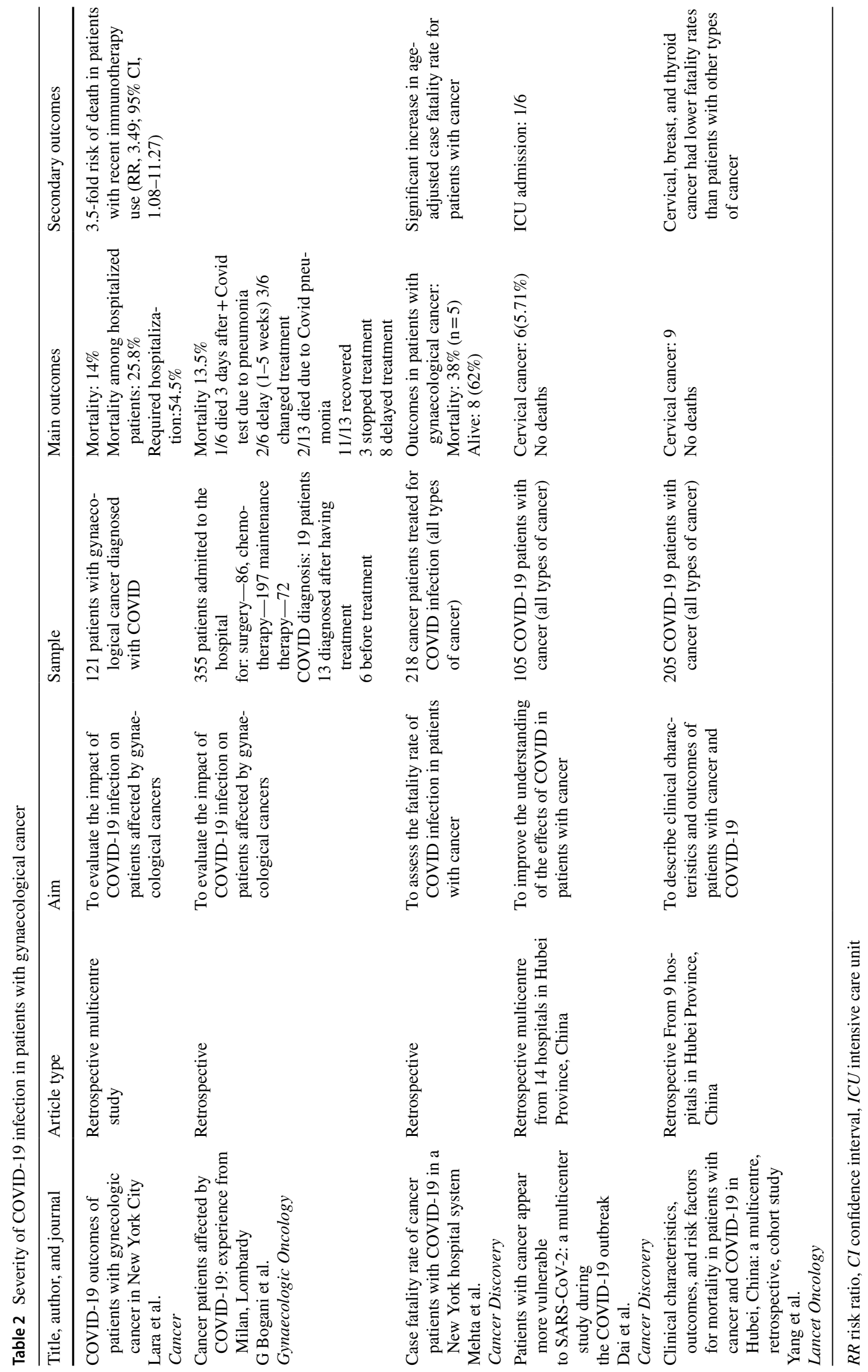


Suh-Burgmann et al. compared the rate of diagnosis of endometrial cancer in California and found a significant change with reduced number of diagnoses and reduced volume of calls about abnormal vaginal bleeding [15].

Significant changes were also observed in a multicenter study in Austria, which included patients with gynaecological and breast cancer. Strong decline in newly diagnosed cancers since the lockdown was noted and half of the patients who were diagnosed with gynaecological cancer in 2020 had no comorbidities, versus 35\% in $2019(p<0.001)$. Choice of primary treatment changed after lockdown in favour of neoadjuvant therapy [16].

In a review of practice from 12 gynecologic cancer centers across Turkey, patients scheduled for surgery were screened for symptoms of COVID-19 in the preoperative period and most were hospitalized $24-48 \mathrm{~h}$ preoperatively. The study suggested that gynecologic cancer surgery can be performed safely when appropriate measures for COVID-19 safety are taken [17].

A single-center retrospective study from Madrid included 126 patients who were scheduled for surgery and suggested that with adequate preventive and protective measures, cancer surgery was possible and did not significantly compromise patients or staff [2].

\section{Patients' perspectives}

The online patient survey by Frey et al., estimated the effect that the COVID-19 pandemic had in the management and the quality of life of patients with diagnosis of ovarian cancer, with $89 \%$ of patients suffering significant anxiety, which is significantly greater that previously reported (57.9\%). The main concern of the patients was reported as acquiring COVID-19 infection, followed by cancer recurrence, safety of family members, access to healthcare and financial implications [18] (Table 4).

The European Society of Gynecological Oncology (ESGO) attempted to identify the patients' perceptions using questionnaires and women were found to be more fearful of cancer progression (70.9\%) than developing Coronavirus disease [19].

\section{Professionals' perspectives}

Three online surveys were identified (Table 5).

Gynaecologic oncology providers from 44 states in America were questioned and the majority reported cancellations and delays in treatment with significant concerns regarding access to supplies and direct impact on patient care [20].

In the survey by Martineli et al., $97.3 \%$ of oncology professionals reported change in clinical practice including $79.1 \%$ that needed to modify treatment [21].
According to an online survey in India, it appeared that the volume of patients in cancer centers had reduced, with risk of patients having delayed diagnosis and presenting at more advanced and potentially incurable stages of disease [22].

\section{Guidelines for management of patients with gynaecological cancer during the COVID-19 pandemic}

Multiple recommendations and guidelines have been published from around the world, with a systematic review by Uwins et al.[23] analyzing and summarizing recommendations from 26 published articles:

Universal rules need to be applied to reduce the infection rate; screening of patients, visitors and staff for COVID19, limited hospital visits, involvement of minimal staffing, safe distancing, personal protection equipment, isolated areas or even isolated hospitals for cancer cases are essential measures.

It was emphasized that cancer surgery, chemotherapy, and radiotherapy should continue while surgery of elective, benign cases should stop. Adaptation of practice depending on the form of treatment chosen is inevitable and prioritization is of paramount importance, however, all the available forms of treatment should be used. Minimal invasive surgery should not be avoided, especially when gastrointestinal tract is not involved.

Well-informed, joint, patient-physician decision-making is critical. Increased morbidity and mortality associated with COVID-19 infection around the time of cancer treatment should be explained and the option of non-surgical treatment or deferring surgery should be given, along with a supplementary consent form. Additionally the potential of worse survival if treatment is delayed should be outlined. Conservative methods to delay treatment of early disease, such as hormonal treatment of endometrial cancer may be considered if needed.

Appropriate protective measures can ensure safety and continuity of high standard of care in patients with gynaecological cancer.

\section{Discussion}

\section{Main findings}

\section{Mortality}

Mortality of COVID-19 infection is higher in patients with gynaecological cancer than in non-cancer patients, with two studies reporting mortality rate of $14 \%[8,12]$ and one reporting a rate of $38 \%$ [13]. In the latter study, which compared mortality among various types of cancer, 


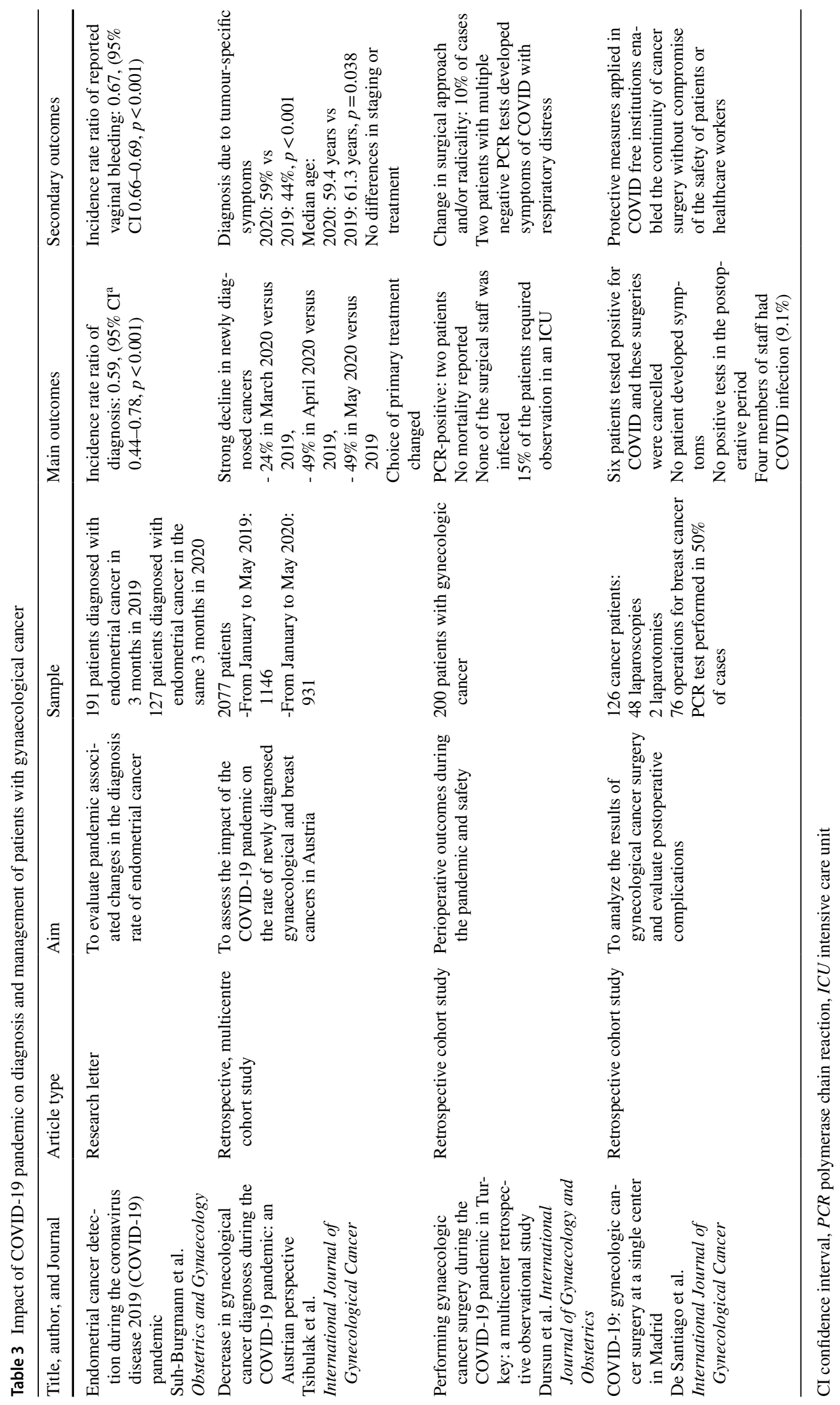




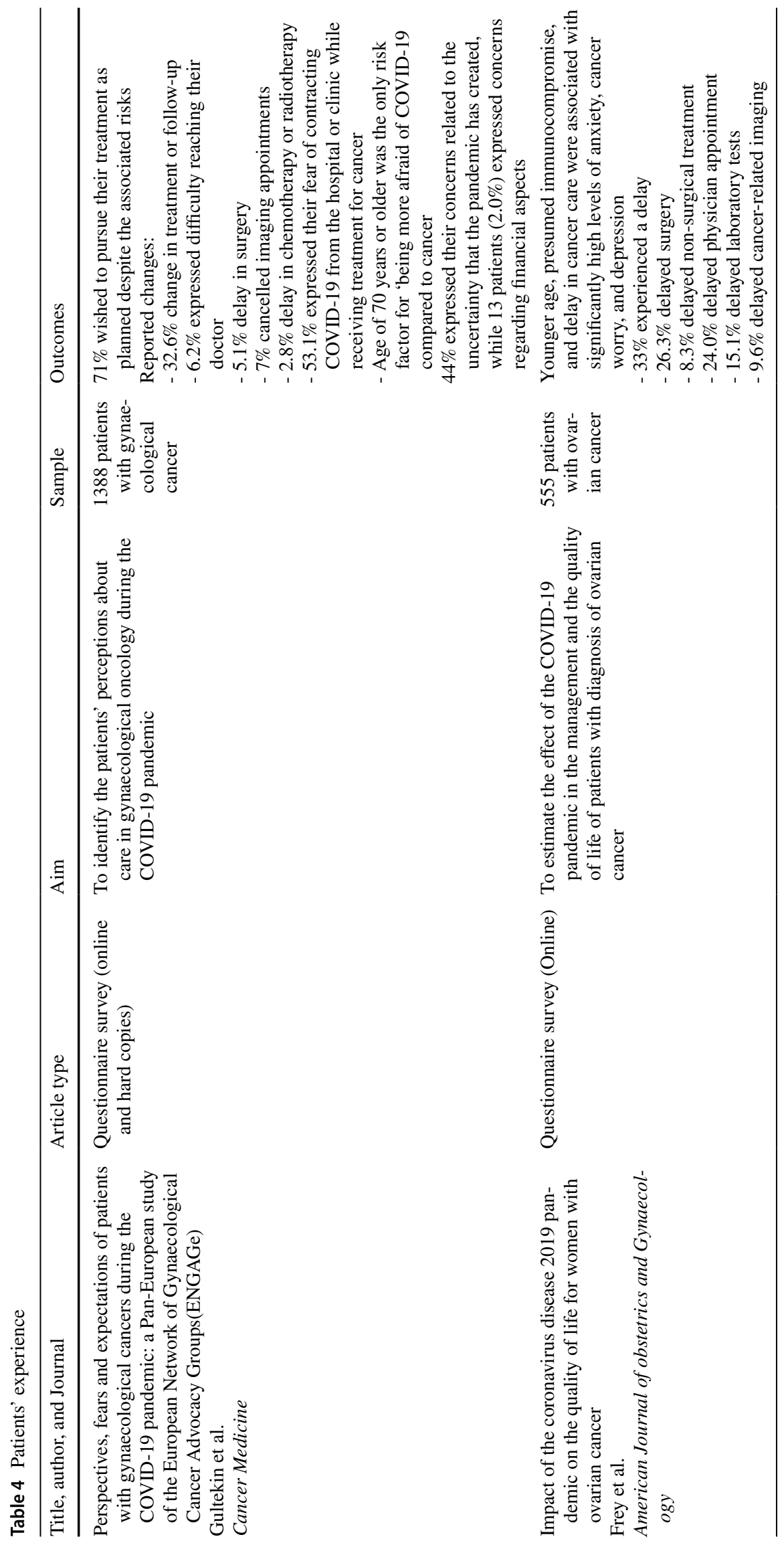




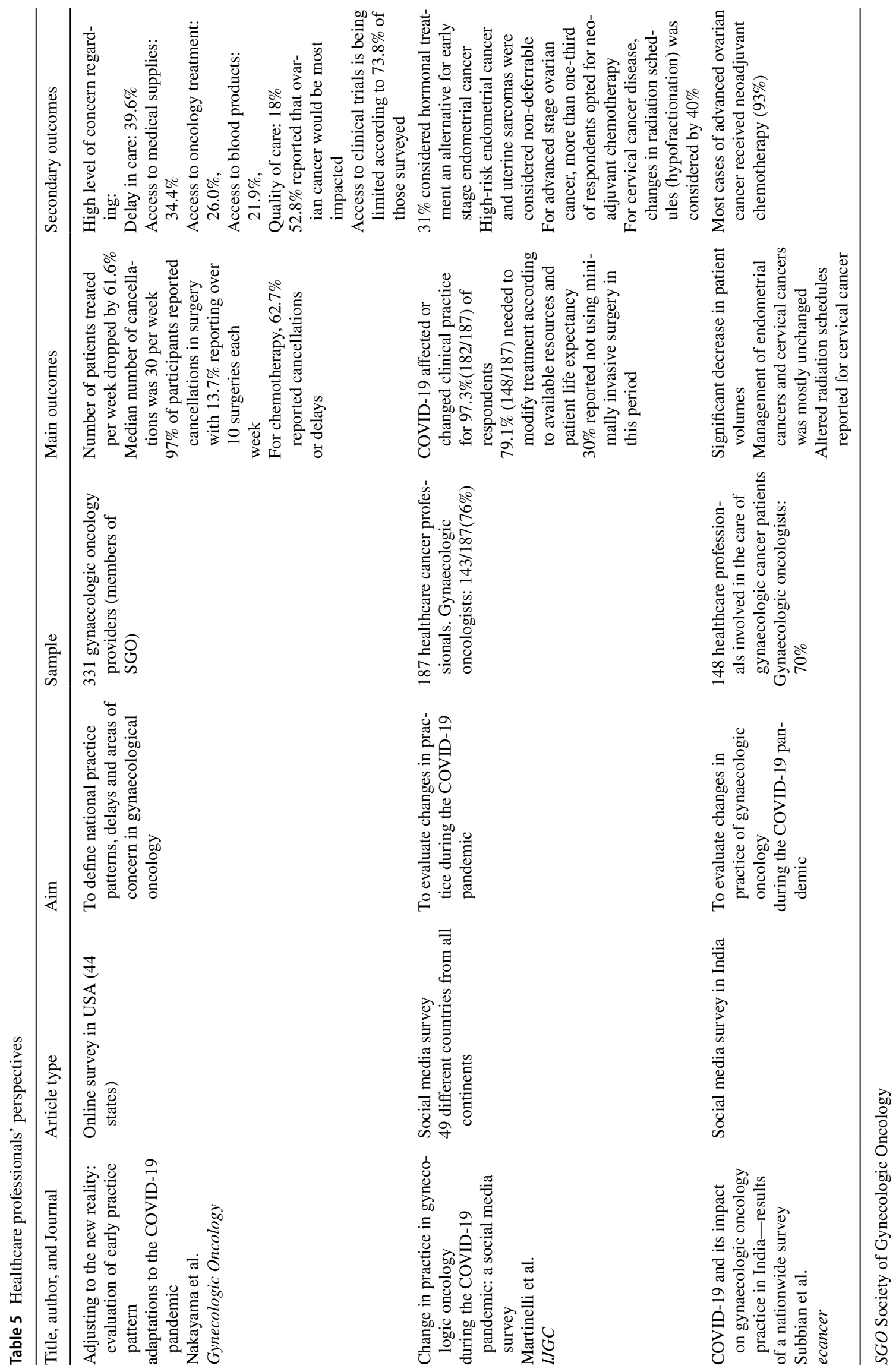


gynaecological patients had the third higher mortality rate after pancreatic and lung cancer.

A nationwide analysis in China, revealed that patients with cancer represented approximately $1 \%$ of the COVID-19 infected population and were considered vulnerable to the infection with a case fatality rate of $5.6 \%$ compared to $2.3 \%$ in the general population [24].

In another large study, history of any cancer was found to be the most significant risk factor for severe events after adjusting for other risk factors including age, comorbidities and history of smoking (odds ratio 5.34, 95\% CI 1.80-16.18; $p=0.0026$ ). Similarly, the risk of rapid deterioration was also greater in those with history of cancer (HR 3.56; 95\% CI 1.65-7.69). Patients who underwent surgery or chemotherapy in the past 1 month had a higher risk of severe events [4].

\section{Cancer care affected by the COVID-19 pandemic}

The vulnerability of cancer patients to a COVID-19 infection and the potential spread of the virus in hospitals, have affected significantly all the areas of medical practice.

Diagnosis of cancer has been affected. In three studies identified, there was a statistically significant reduction in the number of patients diagnosed with gynaecological cancer during the pandemic, comparing to the patients during the same months in 2019 [15-17].

Management has been affected and severe delays in management have been reported. The percentage of the patients experiencing delay in treatment is consistently more than $10 \%$ across the studies identified with most of them being in surgical treatment. There is also a move towards conservative management, with hormonal treatment being utilized in the treatment of endometrial cancer and neoadjuvant chemoradiotherapy being performed in cases, which would be treated with primary surgery before the pandemic. Surgical management has changed with an increased rate of laparotomies compared to laparoscopies despite the preventive measure taken. The consensus from the published articles is that with adequate preventive and protective measures, cancer surgery is possible and does not significantly compromise patients or healthcare workers [2, 15-17].

A recently published systematic review [5], estimated the increase in risk of death after delay in treatment of seven major tumour types: bladder, breast, colon, rectum, lung, cervix, and head and neck, across all three major treatment modalities (surgery, systemic treatment, and radiotherapy). Risk of death is increased after delay of 4 weeks, independent of the treatment modality. For surgery, this is a $6-8 \%$ increase in the risk of death for every 4-week delay. This impact is even more marked for some radiotherapy and systemic indications, with a $13 \%$ increased risk of death for adjuvant systemic treatment for colorectal cancer for example. Specifically for cervical cancer, risk related to a delay in adjuvant radiotherapy was identified, with significant hazard ratio 1.23 (CI 1.00 to 1.50) for each 4-week delay [5]. Another retrospective review of the national cancer database in the United States of more than 200.000 patients, revealed that delay of more than 8 weeks in surgical treatment of low-grade endometrial cancer was independently associated with worsened 5-year survival [25].

Most hospitals globally are avoiding moving patients into the hospital when it is not strictly necessary, and instead are developing teleconsultations and reducing the frequency of outpatient clinic appointments [26]. Several recommendations have been provided to prevent horizontal transmission, requiring patients and healthcare professionals to wear surgical face masks and visors, use alcoholic solutions, reinforce disinfection of surfaces, avoid waiting rooms, and maintain distance when circulating in the ward [24, 27]. Departments are systematically testing asymptomatic patients treatment for COVID-19 infection before treatment or even before clinic appointments [28].

Adapting surgical techniques to reduce the risks of exposure in surgical theatre is recommended. For instance, with open surgery, surgeons attempt to avoid exposure to aerosolized viral particles and reduce operative times. Selfquarantine at home and remote follow-up using teleconsultations for cancer survivors are strongly recommended and are preferred for maintaining regular contacts with patients, postponing non-essential follow-up imaging whenever possible [24].

\section{Views from professionals' perspective}

The overall care has been affected. Results of the surveys are consistent, showing that treatment methods have changed with a move towards conservative and hormonal regimens with neoadjuvant chemotherapy being considered more regularly instead of primary surgery and interval debulking surgery being postponed as much as possible with extended neoadjuvant chemotherapy courses. Radiation schedules have changed with hypofractionation therapy being the preferred method of radiotherapy to avoid multiple hospital attendances.

Severe delays in diagnosis, management and follow-up appointments are the healthcare workers' main concern.

\section{Views from patients' perspective}

The largest patients' survey reported that $71 \%$ of the women asked, would prefer to risk a COVID-19 infection, rather than delay the cancer treatment [19]. However, the pandemic is a cause of severe anxiety, with the majority of patients mentioning fear of acquiring COVID-19 infection and the uncertainty of the disease. Restricted access to healthcare 
and delay in treatment was a cause for Great concern as well $[18,19]$. Similar findings have been documented in other types of cancer [29].

\section{Strengths and limitations}

We followed a standardized and robust systematic rapid review methodology to provide a summary of the way the care of patients with gynaecological cancer has been affected. We reviewed all the available articles, including surveys from healthcare professionals and patients' views, in an attempt to create a comprehensive review regarding all the aspects of gynaecologic oncology.

Our data synthesis may support the efforts for harmonization of guidance and clinical practice and optimization of practice standards internationally.

Nevertheless, studies with relevance may have been omitted, which is an inherent limitation of rapid reviews. There is susceptibility to bias in streamlining a systematic review process, for example in choosing studies for inclusion and in data extraction, as fewer independent reviewers conduct each step.

There is consistency across all studies regarding the way, in which the COVID-19 pandemic has significantly changed practice. This could give the clinicians the insight and the support to make adjustments in every day practice. However, the retrospective nature of the published reviews and the small cohort of patients included in each study unavoidably result in different numeric outcomes and do not allow us to draw definite conclusions.

Therefore, as further evidence emerges and pandemic continues, larger studies are needed and this rapid review could be used as a reference to further research.

\section{Conclusion}

The COVID-19 pandemic has affected clinical practice significantly. Patients with gynaecological cancer and clinicians face changes and challenges in various forms. Diagnoses and treatments have been delayed; different forms of management have been chosen with unknown future risks and women's anxiety has reasonably increased.

Patients with cancer are by definition high risk of contracting COVID-19 infection and developing severe complications. Immunosuppression, frequent hospital appointments and recent treatment, are additional, to the already established, risk factors that could affect this group of patients.

Women with gynaecological cancer have a higher mortality rate than the general population, however, all the studies support the need to avoid delays in diagnosis and treatment, ensuring at the same time application of appropriate protective measures.
Author contributions All authors contributed significantly to the preparation of this manuscript. Professor Doumouchtsis was responsible for the study design and supervision. All authors performed material preparation, data collection, and analysis. Dr. Nikolopoulos wrote the first draft of the manuscript and all authors commented on previous versions of the manuscript. All authors read and approved the final manuscript. Specifically: Dr. MN: protocol/project development, data collection or management, data analysis, manuscript writing/editing. Mr. MM: data analysis, manuscript writing/editing. Prof SD: protocol/ project development, study design, supervision, data collection or management, data analysis, manuscript writing/editing.

Funding The authors have not declared a specific grant for this research from any funding agency in the public, commercial or not-for-profit sectors.

\section{Declarations}

Conflict of interest The authors report no conflict of interest.

\section{References}

1. https://www.who.int/emergencies/diseases/novel-coronavirus2019. World Health Organization. [WHO website]. Accessed 28 Nov 2020.

2. De Santiago J, Yelo C, Chereguini MF et al (2020) COVID-19: gynecologic cancer surgery at a single center in Madrid. Int $\mathrm{J}$ Gynecol Cancer 30(8):1108-1112

3. Ramirez PT, Chiva L, Eriksson AGZ et al (2020) COVID-19 global pandemic: options for management of gynecologic cancers. Int J Gynecol Cancer 30(5):561-563

4. Liang W, Guan W, Chen R et al (2020) Cancer patients in SARSCoV-2 infection: a nationwide analysis in China. Lancet Oncol 21(3):335-337

5. Hanna TP, King WD, Thibodeau S et al (2020) Mortality due to cancer treatment delay: systematic review and meta-analysis. BMJ 371:4087

6. Tricco AC, Lillie E, Straus SE (2017) Rapid review to strengthen health policy and systems: a practical guide. World Health Organization

7. Garritty C, Gartlehner G, Nussbaumer-Streit B et al (2020) Cochrane Rapid Reviews Methods Group offers evidenceinformed guidance to conduct rapid reviews. J Clin Epidemiol 130:13-22

8. Bogani G, Ditto A, Bosio S, Brusadelli C, Raspagliesi F (2020) Cancer patients affected by COVID-19: experience from Milan. Lombardy Gynecol Oncol 158(2):262-265

9. Loganathan J, Doumouchtsis SK (2021) Impact of COVID-19 on management of urogynaecology patients: a rapid review of the literature. Int Urogynecol J 32(10):2631-2646. https://doi.org/10. 1007/s00192-021-04704-2

10. Wells GA, Shea B, O'Connell D, Peterson J, Welch V, Losos M, Tugwell P (2012) The Newcastle-Ottawa Scale (NOS) for assessing the quality if nonrandomized studies in meta-analyses. https:// www.semanticscholar.org/paper/The-Newcastle-Ottawa-Scale(NOS)-for-Assessing-the-Wells-Wells/c293fb316b6176154c3f dbb8340a107d9c8c82bf\#citing-papers. Accessed 30 Nov 2020 
11. Dai M, Liu D, Liu M et al (2020) Patients with cancer appear more vulnerable to SARS-CoV-2: a multicenter study during the COVID-19 outbreak. Cancer Discov 10(6):783-791

12. Lara OD, O'Cearbhaill RE, Smith MJ et al (2020) COVID-19 outcomes of patients with gynecologic cancer in New York City. Cancer 126(19):4294-4303

13. Mehta V, Goel S, Kabarriti R et al (2020) Case fatality rate of cancer patients with COVID-19 in a New York hospital system. Cancer Discov 10(7):935-941

14. Yang K, Sheng Y, Huang C et al (2020) Clinical characteristics, outcomes, and risk factors for mortality in patients with cancer and COVID-19 in Hubei, China: a multicentre, retrospective, cohort study. Lancet Oncol 21(7):904-913

15. Suh-Burgmann EJ, Alavi M, Schmittdiel J (2020) Endometrial cancer detection during the coronavirus disease 2019 (COVID-19) pandemic. Obstet Gynecol 136(4):842-843

16. Tsibulak I, Reiser E, Bogner G et al (2020) Decrease in gynecological cancer diagnoses during the COVID-19 pandemic: an Austrian perspective. Int J Gynecol Cancer 30(11):1667-1671

17. Dursun P, Dervisoglu H, Daggez M et al (2020) Performing gynecologic cancer surgery during the COVID-19 pandemic in Turkey: A multicenter retrospective observational study. Int J Gynaecol Obstet 151(1):33-38

18. Frey MK, Ellis AE, Zeligs K et al (2020) Impact of the coronavirus disease 2019 pandemic on the quality of life for women with ovarian cancer. Am J Obstet Gynecol 223(5):725.e1-e-

19. Gultekin M, Ak S, Ayhan A et al (2020) Perspectives, fears and expectations of patients with gynaecological cancers during the COVID-19 pandemic: A Pan-European study of the European Network of Gynaecological Cancer Advocacy Groups (ENGAGe). Cancer Med 10(1):208-219

20. Nakayama J, El-Nashar SA, Waggoner S, Traughber B, Kesterson $\mathrm{J}$ (2020) Adjusting to the new reality: Evaluation of early practice pattern adaptations to the COVID-19 pandemic. Gynecol Oncol 158(2):256-261
21. Martinelli F, Garbi A (2020) Change in practice in gynecologic oncology during the COVID-19 pandemic: a social media survey. Int J Gynecol Cancer 30(8):1101-1107

22. Subbian A, Kaur S, Patel V, Rajanbabu A (2020) COVID-19 and its impact on gynaecologic oncology practice in India-results of a nationwide survey. Ecancermedicalscience 14:1067

23. Uwins C, Bhandoria GP, Shylasree TS et al (2020) COVID-19 and gynecological cancer: a review of the published guidelines. Int $\mathbf{J}$ Gynecol Cancer 30(9):1424-1433

24. Raymond E, Thieblemont C, Alran S, Faivre S (2020) Impact of the COVID-19 outbreak on the management of patients with cancer. Target Oncol 15(3):249-259

25. Shalowitz DI, Epstein AJ, Buckingham L, Ko EM, Giuntoli RL 2nd (2017) Survival implications of time to surgical treatment of endometrial cancers. Am J Obstet Gynecol 216(3):268.e1-e18

26. Ngoi N, Lim J, Ow S, Jen WY, Lee M, Teo W, Ho J, Sundar R, Tung ML, Lee YM, Ngo E (2020) A segregated-team model to maintain cancer care during the COVID-19 outbreak at an academic center in Singapore. Ann Oncol 31(7):840-843

27. Hellewell J, Abbott S, Gimma A et al (2020) Feasibility of controlling COVID-19 outbreaks by isolation of cases and contacts. Lancet Glob Health 8(4):e488-e496

28. Lambertini M, Toss A, Passaro A et al (2020) Cancer care during the spread of coronavirus disease 2019 (COVID-19) in Italy: young oncologists' perspective. ESMO Open. 5(2):e000759

29. Swainston J, Chapman B, Grunfeld EA, Derakshan N (2020) COVID-19 lockdown and its adverse impact on psychological health in breast cancer. Front Psychol 11:2033

Publisher's Note Springer Nature remains neutral with regard to jurisdictional claims in published maps and institutional affiliations. 